Pacific Journal of Mathematics

COLORINGS OF HYPERMAPS AND A CONJECTURE OF 


\title{
COLORINGS OF HYPERMAPS AND A CONJECTURE OF BRENNER AND LYNDON
}

\section{GiUliana Bianchi AND Robert CORI}

\begin{abstract}
In this paper the following result is obtained: Let $\alpha$ and $\beta$ be two permutations such that $\alpha \beta$ is transitive and $\alpha^{p}=\beta^{q}=1$ (where $p$ and $q$ are distinct primes). Then the set of all permutations commuting both with $\alpha$ and $\beta$ is either reduced to the identity or one of the three cyclic groups $C_{p}, C_{q}$ or $C_{p q}$.
\end{abstract}

Introduction. In this paper we answer a question raised by J. L. Brenner and R. C. Lyndon in [1]. They consider a pair of permutations $(\alpha, \beta)$ acting on a finite set of $n$ elements such that $\alpha^{3}=\beta^{2}=1$ and $\alpha \beta$ is transitive. Such a pair may be considered as a (combinatorial) map with exactly one face in the terminology of [2], [4], [6] and [8], Brenner and Lyndon computed the automorphism group of such a map (which is necessarily a cyclic group) for $n \leq 12$. The groups they find are $1, C_{2}, C_{3}$ and $C_{6}$ and they conjectured that no other groups can arise.

In what follows we prove a more general result and show that if $\alpha \beta$ is transitive and if $p$ and $q$ are primes $(p \neq q)$ such that $\alpha^{p}=\beta^{q}=1$ then the automorphism group of $(\alpha, \beta)$ is one of $1, C_{p}, C_{q}, C_{p q}$. It remains an open question to know whether $C_{p q}$ can be found for arbitrary large values of $n(n \gg p q)$

Our main tool is the introduction of the concept of colorings of a hypermap. These colorings count in a certain way the number of fixed points of an automorphism of $(\alpha, \beta)$ when it acts on the set of cells (i.e. orbits of $\alpha, \beta$ and $\alpha \beta$ ). One step in the proof is to show that an automorphism of prime order cannot have exactly one fixed point in the set of cells: such a result is well known in the theory of Riemann surfaces ([5], p. 266).

All the permutations we consider act on a finite set $\Omega$ of $n$ elements. We will also use the following conventions:

The product $\alpha \beta$ of two permutations $\alpha$ and $\beta$ is the permutation defined by $\alpha \beta(x)=\alpha(\beta(x))$; for a subset $\Omega^{\prime}$ of $\Omega, \alpha \Omega^{\prime}$ denotes the set $\left\{\alpha x \mid x \in \Omega^{\prime}\right\}$, which has the same cardinality as $\Omega^{\prime}$; a permutation $\alpha$ is regular if all its orbits have the same length, which is also the order of $\alpha$; the number of orbits of the permutation $\theta$ will be denoted by $z(\theta)$; a permutation is transitive if $z(\theta)=1$. 
A hypermap is a pair $(\alpha, \beta)$ of permutations such that the group $\langle\alpha, \beta\rangle$ generated by them is transitive on $\Omega$. The orbits of $\alpha, \beta$ and $\alpha \beta$ are the cells of the hypermap.

An automorphism of $(\alpha, \beta)$ is an element $\varphi$ of $\operatorname{Sym}(\Omega)$ that commutes with $\alpha$ and $\beta$. By the transitivity of $\langle\alpha, \beta\rangle$ for any $x$ and $y$ in $\Omega$ there exists $\theta$ in $\langle\alpha, \beta\rangle$ such that $x=\theta y$ and as for any integer $k, \varphi^{k}(x)=$ $\theta \varphi^{k}(x)$ we have

$$
\varphi^{k} x=x \text { if and only if } \varphi^{k} y=y
$$

hence an automorphism of $(\alpha, \beta)$ is a regular permutation.

In order to study the automorphism group of a hypermap we are led to examine for a given permutation $\theta$ the set of regular permutations $\varphi$ commuting with $\theta$. This will be done in detail in the next paragraph.

I. Commuting permutations. We state here for later use some elementary facts about a pair of commuting permutations $\alpha$ and $\beta$ of a finite set. Throughout this section it will be assumed that $\alpha, \beta$ act on a finite set $\Omega$ of $n$ elements and that the group $\langle\alpha, \beta\rangle$ generated by $\alpha$ and $\beta$ is abelian.

We write $\Omega / \alpha$ for the set of $\alpha$-orbits. As $\alpha$ and $\beta$ commute, the actions of $\alpha, \beta$ on $\Omega$ induce actions of $\alpha$ on $\Omega / \beta$ and of $\beta$ on $\Omega / \alpha$.

LEMmA I.1. If $G=\langle\alpha, \beta\rangle$ is transitive, then any element $\theta$ of $G$ is regular.

Proof. For any $x$ and $y$ in $\Omega$ there exists $\varphi$ in $G$ such that $y=\varphi x$, since $\theta^{m} x=x$ and as $\langle\alpha, \beta\rangle$ is abelian, $\theta^{m} y=\varphi \theta^{m} x=y$.

Lemma I.2. If $G=\langle\alpha, \beta\rangle$ is transitive on $\Omega$, then $\alpha$ is transitive on $\Omega / \beta$, and $G$ is also transitive on the set of all intersections $A \cap B$ for $A \in \Omega / \alpha, B \in \Omega / \beta$. Therefore these intersections all have the same cardinality.

Proof. The first statement is clear. If $A, A^{\prime} \in \Omega / \alpha$ and $B, B^{\prime} \in \Omega / \beta$, then $A^{\prime}=\beta^{k} A$ and $B^{\prime}=\alpha^{h} B$ for some $h$ and $k$ in $Z$. Then

$$
\alpha^{h} \beta^{k}(A \cap B)=\alpha^{h}\left(A^{\prime} \cap B\right)=A^{\prime} \cap B^{\prime} .
$$

LEMMA I.3. Let $r$ be the common value of $|A \cap B|, n=|\Omega|$, let $a, b$ be the orders of $\alpha$ and $\beta$. Then there exist $a_{1}, b_{1}$ such that $n=a_{1} b_{1} r, a=a_{1} r$, $b=b_{1} r$. If $b$ is prime then $|\Omega / \alpha|=1$ or $b$. 
Proof. As any $A$ and $B$ are both unions of $A_{i} \cap B_{j}, r$ divides $a$ and $b$, so that $a=a_{1} r, b=b_{1} r$. Since $\alpha$ and $\beta$ are regular $|\Omega / \alpha|=n / a$, $|\Omega / \beta|=n / b$ and there are $n^{2} / a b$ disjoint intersections $A \cap B$. Thus $n=r \cdot\left(n^{2} / a b\right)$ and $n=a b / r=a_{1} b_{1} r$. If $b$ is prime then $r=1$ or $b$ and $n / a=b$ or 1 .

LEMMA I.4. If $\langle\alpha, \beta\rangle$ is transitive, and $a, b, r$ are as above, then there exists an integer $k$ relatively prime with $r$ such that $\alpha^{n / b}=\beta^{n k / a}$.

Proof. Since $\alpha$ is transitive on $\Omega / \beta$, and $|\Omega / \beta|=n / b$ then $\alpha^{n / b}$ stabilizes each $B \in \Omega / \beta$; it also stabilizes each $A \cap B$ as $\alpha A=A$. As $\alpha$ is transitive on $A$ of length $a, \alpha^{n / b}=\alpha^{a / r}$ is transitive on $C=A \cap B$. Similarly $\beta^{n / a}$ is transitive on $C$. For a particular $C$ the restrictions of $\alpha^{n / b}$ and $\beta^{n / a}$ to $C$ generate the same cyclic group of order $r$, then for some $k$ such that $(k, r)=1, \alpha^{n / b}$ and $\beta^{n K / a}$ have the same action on $C$. Thus the element $\alpha^{n / b} \beta^{-n k / a}$ of $\langle\alpha, \beta\rangle$ has at least one fixed point by I.1, it is the identity.

II. Colorings. Throughout this section we assume that $\varphi$ is a regular permutation of order $m$ acting on a finite set $\Omega$ of $n$ elements.

A coloring on the set $\Omega$ is a map $\lambda$ defined on $\Omega$ with values in an abelian group $R$. For any permutation $\alpha$ and any coloring $\lambda$ of $\Omega$ we define another coloring $D_{\alpha} \lambda$ by setting

$$
D_{\alpha} \lambda(x)=\lambda(\alpha(x))-\lambda(x) .
$$

A coloring is said to be orthogonal to $\alpha$ if $D_{\alpha} \lambda$ is constant on $\Omega$. In this case $\lambda\left(\alpha^{k}(x)\right)=\lambda(x)+k \cdot u$ where $u$ is the constant value of $D_{\alpha} \lambda$. The length $l$ of an orbit of $\alpha$ must verify $l u=0$ in the abelian group. As we will only consider colorings orthogonal to $\varphi$, we will assume that $R$ is the additive group $Z / m Z$. Thus the relation $m u=0$ is satisfied for any $u$.

We are now interested in the extension of a coloring vanishing on a transversal $T$ of $\Omega / \varphi$, and having a given value $v$ on an element $x$ not in $T$. For such an $x$ there exists a unique $\bar{x}$ in $T$ and an integer $h(1 \leq h \leq m)$ such that $\varphi^{h}(\bar{x})=x$.

LEMMA II.1. For $v$ in $Z / m Z$, there exists a coloring $\lambda$ orthogonal to $\varphi$, vanishing on $T$ and such that $\lambda(x)=v$ if and only if the equation in $u$, $h u \equiv v$, has a solution in $Z / m Z$.

Proof. If $D_{\alpha} \lambda$ is a constant $u$, then $\lambda(x)=\lambda(\bar{x})+h u$ so that $h u=v$. If this equation has a solution $u_{0}$ say, then for any $y$ in $\Omega$ there exists $\bar{y}$ in $T$ such that $y=\varphi^{\prime}(\bar{y})$; setting $\lambda(y)=l u_{0}$ we obtain the coloring $\lambda$. 
LEMMA II.2. Let $\langle\varphi, \alpha\rangle$ be abelian and $\lambda$ be a coloring orthogonal to $\varphi$. Then $D_{\alpha} \lambda$ is constant on the orbits of $\varphi$.

Proof. We have to show that $D_{\alpha} \lambda(\varphi x)=D_{\alpha} \lambda(x)$. But as $D_{\alpha} \lambda(\varphi(x))$ $=\lambda \alpha \varphi x-\lambda \varphi x$ and since $\alpha$ and $\varphi$ commute:

$$
\begin{aligned}
D_{\alpha} \lambda \varphi(x) & =\lambda \varphi \alpha x-\lambda \alpha x+\lambda \alpha x-\lambda x+\lambda x-\lambda \varphi x \\
& =D_{\varphi} \lambda(\alpha x)+D_{\alpha} \lambda(x)-D_{\varphi} \lambda(x) .
\end{aligned}
$$

As $D_{\varphi} \lambda$ is constant, also the result follows. Remark that $D_{\alpha} \lambda$ defines a coloring on $\Omega / \varphi$. For $A$ in $\Omega / \varphi, D_{\alpha} \lambda(A)$ denotes the common value of $D_{\alpha} \lambda(x)$ for $x$ in $A$.

LEMmA II.3. Let $\langle\varphi, \alpha\rangle$ be abelian and transitive on $\Omega$. Then there exists a coloring $\lambda$ orthogonal to $\varphi$, such that

$$
\sum_{A \in \Omega / \varphi} D_{\alpha} \lambda(A) \equiv z(\alpha) \text { in } Z / m Z
$$

Proof. Let $|\Omega|=n, \alpha$ have order $a$, and let $r$ be the cardinality of the intersection of an orbit of $\alpha$ with one of $\varphi$. As $\alpha$ is transitive on $\Omega / \varphi$ there exists $x$ such that $T=\left\{x, \alpha x, \ldots, \alpha^{n / m-1} x\right\}$ is a transversal of $\Omega / \varphi$. Let $y=\alpha^{n / m} x$; we claim that there exists $\lambda$ vanishing on $T$ and such that $\lambda(y)=z(\alpha)=n / a$.

By Lemma I.4 there exists $k$ such that $\varphi^{n / a \cdot k}=\alpha^{n / m}$; then $y=$ $\varphi^{n / a \cdot k}(x)$. By II.1 such a $\lambda$ exists if the equation

$$
n k u / a \equiv n / a
$$

has a solution in $Z / m Z$.

But since $(k, r)=1$ there exist $u, v$, such that $u k+v r=1$. Then

$$
n k u / a+n v r / a=n / a
$$

and as $n r / a=m(\mathrm{I} .3)$, we are done.

Lemma II.4. Let $G=\langle\varphi, \alpha\rangle$ be abelian. Then there exists a coloring $\lambda$ such that $D_{\varphi} \lambda$ is constant on G-orbits and such that

$$
\sum_{A \in \Omega / \varphi} D_{\alpha} \lambda(A)=z(\alpha)
$$

Moreover if $\varphi$ is of prime order and fixes only one orbit of $\alpha$ then $\lambda$ can be found orthogonal to $\varphi$. 
Proof. By Lemma II.3 for any $G$-orbit $\Omega_{h}$ there exists a coloring $\lambda_{h}$ such that $D_{\varphi} \lambda_{h}$ is constant on $\Omega_{h}$ and

$$
\sum_{A \in \Omega_{h} / \varphi} D_{\alpha} \lambda_{h}(A)=z\left(\alpha_{h}\right)
$$

where $\alpha_{h}$ is the restriction of $\alpha$ to $\Omega_{h}$. Taking for $\lambda$ the union of the $\lambda_{h}$ we have the result, since $z(\alpha)=\Sigma z\left(\alpha_{h}\right)$. If $\varphi$ is of prime order, then by I.3 $\left|\Omega_{h} / \alpha\right|=1$ or $m$. In the first case, $\Omega_{h}$ is an $\alpha$ orbit fixed by $\varphi$. This occurs only once, for $h_{0}$ say; the equation to solve in $\Omega_{h_{0}}$ is $k u \equiv 1(\bmod m)$ which gives $u=k^{-1}$ in $Z / m Z$. In the second case $\left|\Omega_{h} / \alpha\right|=m$ and the equation to solve is $m k^{\prime} u \equiv m(\bmod m)$ which is satisfied by any $u$, in particular for $u=k^{-1}$. We thus can choose $\lambda$ such that $D_{\alpha} \lambda=u$ on any $\Omega_{h} \cdot D_{\alpha} \lambda$ is thus constant.

LEMma II.5. Let $G=\langle\varphi, \alpha\rangle$ be abelian and such that the intersection $A_{i} \cap B_{j}$ of an orbit of $\varphi$ with one of $\alpha$ contains at most one element. Then for any coloring $\lambda$ orthogonal to $\varphi$ we have

$$
\sum_{A \in \Omega / \varphi} D_{\alpha} \lambda(A)=0 .
$$

Proof. It suffices to show that the sum vanishes on each $\alpha$ orbit in $\Omega / \varphi$. Let $C$ be such an orbit; under the hypothesis of the lemma, there exists an orbit $\Gamma$ in $\Omega$ of length $|C|$ and $\Sigma_{c \in C} D_{\alpha} \lambda(c)=\Sigma_{x \in \Gamma} D_{\alpha} \lambda(x)$. But $\Gamma=\left\{x, \alpha x, \ldots, \alpha^{k} x\right\}$ and the last sum is $\sum_{i=0}^{k}\left(\lambda \alpha^{i+1} x-\lambda \alpha^{i} x\right)$ which vanishes as $\alpha^{k+1} x=x$.

LEMMA II.6. Let $\alpha$ and $\beta$ be any two permutations commuting with $\varphi$, then for any coloring $\lambda$ orthogonal to $\varphi$ one has

$$
\sum_{A \in \Omega / \varphi} D_{\alpha \beta} \lambda(A)=\sum_{A \in \Omega / \varphi} D_{\alpha} \lambda(A)+\sum_{A \in \Omega / \varphi} D_{\beta} \lambda(A) .
$$

Let $\Gamma$ be any subset of $\Omega$ having exactly one element in each cycle of $\varphi$. Then

$$
\sum_{A \in \Omega / \varphi} D_{\alpha \beta} \lambda(A)=\sum_{x \in \Gamma} D_{\alpha \beta} \lambda(x) .
$$

Since $D_{\alpha \beta} \lambda(a)=\lambda(\alpha \beta(a))-\lambda(\beta(a))+\lambda(\beta(a))-\lambda(a)$ we have

$$
\sum_{A \in \Omega / \varphi} D_{\alpha \beta} \lambda(A)=\sum_{x^{\prime} \in \beta(\Gamma)} D_{\alpha} \lambda\left(x^{\prime}\right)+\sum_{x \in \gamma} D_{\beta} \lambda(x) .
$$

But $\beta(\Gamma)$ is also a subset of $\Omega$ having one element in each cycle of $\varphi$ and the result follows. 


\section{The main theorems.}

THEOREM 1. Let $H=(\alpha, \beta)$ be a hypermap $\varphi$ an automorphism of $H$ of prime order $p$. Then the number of cells fixed by $\varphi$ is necessarily different from one.

Proof. Let us show that the assumption that $\varphi$ fixes exactly one cell leads to a contradiction. Suppose that this cell is an orbit of $\alpha$ (a similar proof holds for an orbit of $\beta$ or $\alpha \beta$ ). If $\varphi$ fixes no other cycle of $\alpha$ then $z(\alpha)-1$ is clearly divisible by $p$. Then by Lemma II.4 there exists a coloring orthogonal to $\varphi$ such that $\Sigma_{A \in \Omega / \varphi} D_{\alpha} \lambda(A) \equiv 1(\bmod p)$, but by Lemma II.6.

$$
\sum_{A \in \Omega / \varphi} D_{\alpha \beta} \lambda(A)=\sum_{A \in \Omega / \varphi} D_{\alpha} \lambda(A)+\sum_{A \in \Omega / \varphi} D_{\beta} \lambda(A)
$$

and Lemma II.5 insures the nullity of $\Sigma_{A \in \Omega / \varphi} D_{\alpha \beta} \lambda(A)$ and $\sum_{A \in \Omega / \varphi} D_{\beta} \lambda(A)$. As no cycle of either $\beta$ or $\alpha \beta$ is fixed by $\varphi$, we have thus found a contradiction and Theorem II.1 is proved.

LEMMA III. 1 Let $\varphi$ be a permutation of order $p^{2}$ commuting with $\alpha$ of order $p$. Then for any $\lambda$ orthogonal to $\varphi$

$$
p \sum_{A \in \Omega / \varphi} D_{\alpha} \lambda(A) \equiv 0 \quad\left(\bmod p^{2}\right) .
$$

Proof. We can assume that $\langle\varphi, \alpha\rangle$ acts transitively on $\Omega$; the general case is then obtained by summing over the orbits of $\langle\varphi, \alpha\rangle$.

Since $\alpha$ is of order $p$, by Lemma I.3 the cardinality of the intersection of a cycle of $\varphi$ and one of $\alpha$ is either 1 or $p$. If it is 1 , then by Lemma II.4 we have

$$
\sum_{A \in \Omega / \varphi} D_{\alpha} \lambda(A) \equiv 0 \quad\left(\bmod p^{2}\right)
$$

If it is $p$, then $\Omega / \varphi$ has only one element. Let $\varphi=\left(b_{1}, b_{2}, \ldots, b_{p^{2}}\right)$. The $\operatorname{sum} \Sigma_{A \in \Omega / \varphi} D_{\alpha} \lambda(A)$ equals $D_{\alpha} \lambda\left(b_{1}\right)$ and we find

$$
D_{\alpha} \lambda\left(b_{1}\right)=\lambda\left(\alpha\left(b_{1}\right)\right)-\lambda\left(b_{1}\right) \text {. }
$$

But as $\varphi$ and $\alpha$ commute and $\varphi$ is a cycle, $\alpha$ is a power of $\varphi$ and $\alpha=\varphi^{i p}$, $0 \leq i \leq p-1$. Thus as $\lambda$ is orthogonal to $\varphi, \lambda\left(\alpha\left(b_{1}\right)\right)=\lambda\left(\varphi^{i p}\left(b_{1}\right)\right)=$ $\lambda\left(b_{1}\right)+i p u$, so that $D_{\alpha} \lambda\left(b_{1}\right)=i p u$, as required.

We are now able to prove our main theorem. 
TheOREM 2. Let $p$ and $q$ be two distinct primes $\alpha$ and $\beta$ be two permutations such that

(1) $\alpha \beta$ is a cycle,

(2) $\alpha^{q}=\beta^{p}=1$.

Then the automorphism group of $(\alpha, \beta)$ is either trivial or one of $C_{p}, C_{q}, C_{p q}$.

Proof. It is clear that Aut $\langle\alpha, \beta\rangle$ is cyclic.

Let now $\varphi$ be an automorphism of prime order, clearly $\varphi$ fixes one cell of the hypermap $(\alpha, \beta)$ : the unique cycle of $\alpha \beta$. By Theorem 1 it fixes one more cell, if this cell is of length one then $\varphi$ is the identity, if it is of length $p$ or $q$ then clearly $\varphi$ has orbits of length dividing $p$ or $q$ and $\varphi$ is of order $p, q$ or 1 . This proves that $\operatorname{Aut}\langle\alpha, \beta\rangle$ is of order $p^{u} q^{v}$. To obtain the complete result we will show that assuming the existence of an automorphism of order $m=p^{2}$ (or $m=q^{2}$ similarly) we have a contradiction. Let $\varphi$ be such an automorphism, let $\lambda$ be the coloring constructed in Lemma II. 3 for $\alpha \beta$, we have

$$
\sum_{A \in \Omega / \varphi} D_{\alpha \beta} \lambda(A) \equiv z(\alpha \beta) \quad(\equiv 1)\left(\bmod p^{2}\right) .
$$

But by Lemma II.6:

$$
1 \equiv \sum_{A \in \Omega / \varphi} D_{\alpha \beta} \lambda(A) \equiv \sum_{A \in \Omega / \varphi} D_{\alpha} \lambda(A)+\sum_{A \in \Omega / \varphi} D_{\beta} \lambda(A) \quad\left(\bmod p^{2}\right)
$$

and

$$
\sum_{A \in \Omega / \varphi} D_{\beta} \lambda(A) \equiv 0 \quad\left(\bmod p^{2}\right)
$$

as the cardinality $r$ of the intersection of a cycle of $\varphi$ and one of $\beta$ is 0 or 1 ( $r$ dividing $p^{2}$ and $q$ ).

We thus have using Lemma III.1 and multiplying by $p$ the above equality:

$$
p \equiv p \sum_{A \in \Omega / \varphi} D_{\alpha} \lambda(A) \equiv 0 \quad\left(\bmod p^{2}\right)
$$

Which is the contradiction we are looking for.

Acknowledgement. The authors wish to thank R. C. Lyndon for many helpful comments on a preliminary version of this work. 


\section{REFERENCES}

[1] J. L. Brenner and R. C. Lyndon, Permutations and cubic graphs, Pacific J. Math., 104 (1983), 285-315.

[2] R. Cori, Un code pour les graphes planaires et ses applications, Astérisque, Société Mathématique de France, 27 (1975).

[3] R. Cori, A. Machí, J. G. Penaud and B. Vauquelin, On the automorphism group of a planar hypermap, European J. Combin., 2 (1981), 331-334.

[4] J. Edmonds, A combinatorial representation for polyhedral surfaces, Notices Amer. Math. Soc., 7 (1960), 646.

[5] H. M. Farkas and I. Kra, Riemann Surfaces, Springer-Verlag (1980).

[6] A. Jacques, Sur le genre d'une paire de substitutions, C. R. Acad. Sci. Paris, série A.B, 267 (1968), 625-627.

[7] V. A. Liskovets, $A$ census of non isomorphic planar maps, Colloquia Matematica Societatis János Bolyai (1-25), (1978)-Algebraic method in graph theory, p. 479-494.

[8] T. R. S. Walsh, Hypermap versus bipartite maps, J. Combinatorial Theory-Ser. B, 18 (1975), 155-163.

Received December 9, 1981 and in revised form May 24, 1982.

UNIVERSITY OF BORDEAUX I

351, COURS DE LA LIBERATION

33405 Talence Cedex, France 


\section{PACIFIC JOURNAL OF MATHEMATICS \\ EDITORS}

DONALD BABBITT (Managing Editor)

University of California

Los Angeles, CA 90024

Hugo RossI

University of Utah

Salt Lake City, UT 84112

C. C. MOOre and ARThur OGus

University of California

Berkeley, CA 94720
J. Dugundi

Department of Mathematics

University of Southern California

Los Angeles, CA 90089-1113

R. FINN and H. SAMELSON

Stanford University

Stanford, CA 94305

ASSOCIATE EDITORS

$\begin{array}{lllll}\text { R. Arens } & \text { E. F. Beckenbach } & \text { B. H. Neumann } & \text { F. Wolf } & \text { K. Yoshida }\end{array}$ $(1906-1982)$

\section{SUPPORTING INSTITUTIONS}

UNIVERSITY OF ARIZONA

UNIVERSITY OF BRITISH COLUMBIA

CALIFORNIA INSTITUTE OF TECHNOLOGY

UNIVERSITY OF CALIFORNIA

MONTANA STATE UNIVERSITY

UNIVERSITY OF NEVADA, RENO

NEW MEXICO STATE UNIVERSITY

OREGON STATE UNIVERSITY
UNIVERSITY OF OREGON

UNIVERSITY OF SOUTHERN CALIFORNIA

STANFORD UNIVERSITY

UNIVERSITY OF HAWAII

UNIVERSITY OF TOKYO

UNIVERSITY OF UTAH

WASHINGTON STATE UNIVERSITY

UNIVERSITY OF WASHINGTON 


\section{Pacific Journal of Mathematics}

\section{Vol. 110, No. $1 \quad$ September, 1984}

Wojciech Abramczuk, A class of surjective convolution operators $\ldots \ldots \ldots \ldots 1$

K. Adachi, Extending bounded holomorphic functions from certain

subvarieties of a weakly pseudoconvex domain $\ldots \ldots \ldots \ldots \ldots \ldots$

Malvina Florica Baica, An algorithm in a complex field and its application

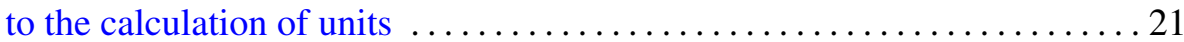

Giuliana Bianchi and Robert Cori, Colorings of hypermaps and a

conjecture of Brenner and Lyndon $\ldots \ldots \ldots \ldots \ldots \ldots \ldots \ldots \ldots \ldots \ldots \ldots$

Ronald James Evans, Determinations of Jacobsthal sums . . .......... 49

Leslie Foged, Characterizations of $\aleph$-spaces .................... 59

Nassif A. Ghoussoub and Paulette Saab, Weak compactness in spaces of

Bochner integrable functions and the Radon-Nikodým property . . . . . . 65

J. Gómez Gil, On local convexity of bounded weak topologies on Banach

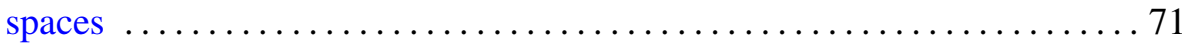

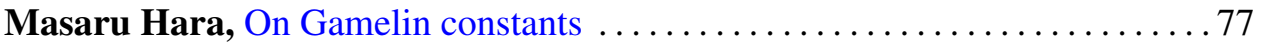

Wilfried Hauenschild, Eberhard Kaniuth and Ajay Kumar, Harmonic

analysis on central hypergroups and induced representations $\ldots \ldots \ldots 83$

Eugenio Hernandez, An interpolation theorem for analytic families of

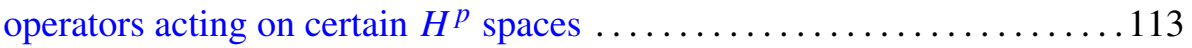

Thomas Alan Keagy, On "Tauberian theorems via block-dominated

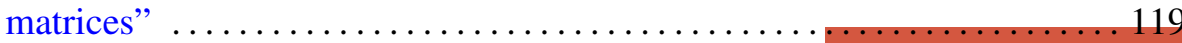

Thomas Landes, Permanence properties of normal structure $\ldots \ldots \ldots \ldots \ldots 125$

Daniel Henry Luecking, Closed ranged restriction operators on weighted

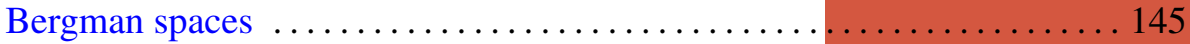

Albert Thomas Lundell, The $p$-equivalence of $\mathrm{SO}(2 n+1)$ and $\mathrm{Sp}(n) \ldots \ldots 161$

Mark D. Meyerson, Remarks on Fenn's "the table theorem" and Zaks' "the chair theorem" ..................................... 167

Marvin Victor Mielke, Homotopically trivial toposes . . . . . . . . . . 171

Gerard J. Murphy, Hyperinvariant subspaces and the topology on Lat A . . 183

Subhashis Nag, On the holomorphy of maps from a complex to a real manifold

Edgar Milan Palmer and Robert William Robinson, Enumeration of self-dual configurations ................................. 203

John J. Walsh and David Clifford Wilson, Continuous decompositions

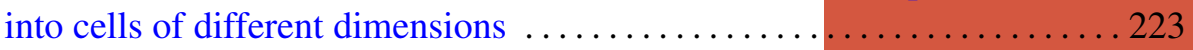

Walter John Whiteley, Infinitesimal motions of a bipartite framework .....233 